\title{
Laser photoablation: a new biomedical tool
}

Nozomi Nishimura, Chris Schaffer, Beth Friedman, Philbert Tsai, Patrick Lyden, and David Kleinfeld

Ultrashort laser pulses can be used to produce lesions in single blood vessels located in the cortex of live rats, thus enabling the study of microstrokes.

Most diseases and biological processes can only be studied in a living animal in which all the complex regulatory processes are occurring. ${ }^{1}$ New techniques using ultrashort laser pulses are increasingly allowing researchers to manipulate cells and tissue within living organisms to study normal and diseased functionality. The study of microstrokes may, in particular, benefit from these advances. These small strokes are thought to be caused by clots or bleeding in small blood vessels within the brain that lead to the death of neurons. Clinical studies have shown that such microstrokes are relatively common in the elderly and may play a major role in dementia and diseases such as Alzheimer's. ${ }^{2}$ However, no treatment is presently available, partly because reproducing microstroke lesions in animals has proved elusive. Ultrashort pulsed laser ablation provides a novel tool to produce such lesions, finally enabling the study of this disease. ${ }^{3}$

The technique has recently emerged as an excellent tool for the precision machining of solid state materials ${ }^{4-6}$ and has been used for the fabrication of waveguides ${ }^{7}$ inside transparent materials without affecting the surface. The extremely high light intensities achieved when focusing ultrashort laser pulses enable the nonlinear optical absorption of the laser light. The resulting ionized volume can be as small as the size of a diffraction-limited focus, typically $\sim 1 \mu \mathrm{m}^{3}$, and can be confined deep below the surface. This same technique can be applied to manipulating biological structures deep within a living organism.

To study the effect of microstrokes in the living brain, windows are made in the skulls of anesthetized rats and the blood vessels are labeled with an intravenous injection of fluorescent dye. We use another nonlinear optical technique, two-photon laser scanning microscopy, to image blood vessels and measure blood flow in the brain with microscopic resolution. To perturb microvessels located as deep as $500 \mu \mathrm{m}$ below the brain surface,
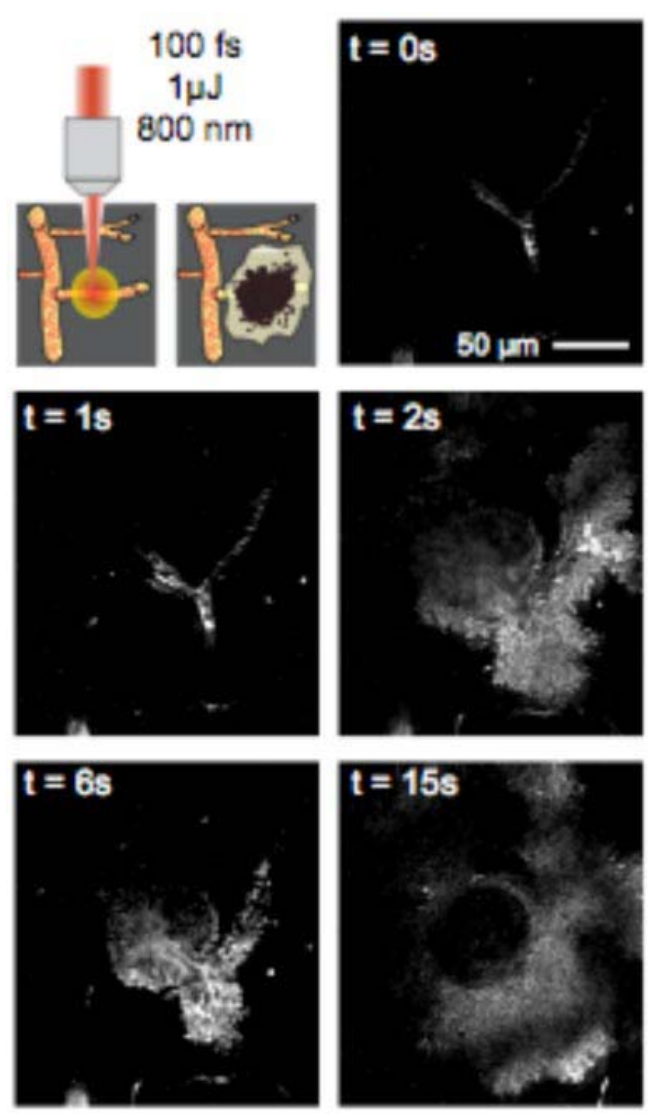

Figure 1. Diagram of the femtosecond laser-induced rupture of a microvessel followed by hemorrhage (top, left). The other panels show the time series of five images showing the rupture of a vessel $140 \mu \mathrm{m}$ below the surface of a rat cortex. Each image is labelled with the time relative to irradiation onset. The images were recorded during two-photon excited fluorescence microscopy. The targeted vessel first explodes, leaving a dark central volume filled with red blood cells and fluorescentlylabeled blood plasma penetrating the brain tissue. The illustrations are adapted from Nishimura et al. ${ }^{3}$ and used with kind permission of the Nature Publishing Group. 
laser pulses $(800 \mathrm{~nm}, 100 \mathrm{fs}, \sim 0.01-5 \mu \mathrm{J})$ from an amplified titanium:sapphire laser are focused on the center of the target blood vessel. ${ }^{3}$ At the lowest energies, a train of pulses can be used to cause the vessel to leak blood into the surrounding brain tissue. Some blood proteins are neurotoxic and may cause microstrokes. Further irradiation of a leaky vessel can trigger natural clotting and block the vessel. Lack of blood flow is another route by which strokes can occur because neurons survive only a short time in the absence of blood flow. At the higher energy range, the target vessel is ruptured and bleeds into the surrounding brain tissue, forming a hemorrhage (see Figure 1). Similar hemorrhages appear to have been found in human patients. ${ }^{8}$

Using two-photon microscopy we observed that the blood flow in vessels close to and downstream from the clot was severely reduced. This is in stark contrast with results obtained for clotting arterioles (narrow blood vessels that branch art from arteries) on the surface of the brain, recently shown not to cause severe blood flow reductions. ${ }^{9}$ Our result may appear counterintuitive because surface arterioles, being much larger than deep microvessels, would lead one to expect their clotting to have a greater effect on the blood than a clot in a fine subsurface vessel. But if we consider that surface arteriole networks have loops that protect against blood flow decreases due to single-vessel clots, our result become understandable.

We also investigated therapeutic strategies—such as lowering blood viscosity - to allay the effects of microstrokes. To reduce the effective blood viscosity, we intravenously injected a rat with a large amount of saline after induction of a microvessel clot, essentially diluting the blood in order to make it flow more easily. Blood flow as close as two branches away from the clot showed substantial recovery after treatment, which suggests that drugs that manipulate blood viscosity might be useful in treating patients.

Ultrashort pulsed laser ablation thus represents a tool with sufficiently fine spatial resolution to selectively target cells and structures of interest. Since it can be used in live animals, it has great potential for the study of diseases such as microstrokes and other biological phenomena. The usefulness of microscopic manipulation in whole animals has also been demonstrated in neuroscience studies in which neuronal processes were severed to study their effect on behavior. ${ }^{10,11}$ Such applications demonstrate that there are many opportunities for advanced optical technologies to contribute in many areas of biology.

\section{Author Information}

\section{Nozomi Nishimura}

Department of Biomedical Engineering

Cornell University

Ithaca, NY

Department of Physics
University of California at San Diego

La Jolla, CA

http:/ / www.bme.cornell.edu/schafferlab

\section{Chris Schaffer}

Department of Biomedical Engineering

Cornell University

Ithaca, NY

http:/ / www.bme.cornell.edu/schafferlab

\section{Beth Friedman}

Department of Neurosciences

University of California at San Diego

La Jolla, CA

\section{Philbert Tsai}

Department of Physics

University of California at San Diego

La Jolla, CA

\section{Patrick Lyden}

Department of Neurosciences and Graduate Program in Neurosciences

University of California at San Diego

La Jolla, CA

\section{David Kleinfeld}

Department of Physics, Center for Theoretical Biological Physics and Graduate Program in Neurosciences

University of California at San Diego

La Jolla, CA

\section{References}

1. T. Misgeld and M. Kerschensteiner, In vivo imaging of the diseased nervous system, Nat. Rev. Neurosci. 7, pp. 449-463, 2006.

2. J. T. O’Brien, T. Erkinjuntti, B. Reisberg, G. Roman, T. Sawada, L. Pantoni, J. V. Bowler, C. Ballard, C. DeCarli, P. B. Gorelick, K. Rockwood, A. Burns, S. Gauthier, and S. T. DeKosky, Vascular cognitive impairment, Lancet Neurol 2, pp. 89-98, 2003. 3. N. Nishimura, C. B. Schaffer, B. Friedman, P. S. Tsai, P. D. Lyden, and D. Kleinfeld, Targeted insult to subsurface cortical blood vessels using ultrashort laser pulses: three models of stroke, Nat. Methods 3, pp. 99-108, 2006.

4. A. P. Joglekar, H. H. Liu, E. Meyhofer, G. Mourou, and A. J. Hunt, Optics at critical intensity: Applications to nanomorphing, Proc. Nat'1 Acad. Sci. USA 101, pp. 5856$5861,2004$.

5. S. Nolte, G. Kamlage, F. Korte, T. Bauer, T. Wagner, A. Ostendorf, C. Fallnich, and H. Welling, Microstructuring with femtosecond lasers, Adv. Eng. Materials 2, pp. 2327, 2000.

6. C. B. Schaffer, A. Brodeur, and E. Mazur, Laser-induced breakdown and damage in bulk transparent materials induced by tightly-focused femtosecond laser pulses, Meas. Sci. Tech. 12, pp. $1784-1794,2001$.

7. C. B. Schaffer, A. Brodeur, J. F. Garcia, and E. Mazur, Micromachining bulk glass by use of femtosecond laser pulses with nanojoule energy, Opt. Lett. 26, pp. 93-95, 2001.

8. K. M. Cullen, Z. Kocsi, and J. Stone, Pericapillary haem-rich deposits: evidence for microhaemorrhages in aging human cerebral cortex, J. Cereb. Blood Flow Metab. 25, pp. 1656-1667, 2005. 
9. C. B. Schaffer, B. Friedman, N. Nishimura, L. F. Schroeder, P. S. Tsai, F. F. Ebner, P. D. Lyden, and D. Kleinfeld, Two-photon imaging of cortical surface microvessels reveals a robust redistribution in blood flow after vascular occlusion, Pub. Lib. of Sci. (PLoS) Biology 4, p. e22, 2006.

10. S. H. Chung, D. A. Clark, C. V. Gabel, E. Mazur, and A. D. Samuel, The role of the AFD neuron in C. elegans thermotaxis analyzed using femtosecond laser ablation, BMC Neurosci 7, p. 30, 2006

11. M. F. Yanik, H. Cinar, H. N. Cinar, A. D. Chisholm, Y. Jin, and A. Ben-Yakar,

Neurosurgery: functional regeneration after laser axotomy, Nature 432, p. 822, 2004. 